\title{
Voluntary Thigh Muscle Strength with Resection Stump-Dependent Blood Flow and Vasodilation in an Amputated Lower Leg with Total Surface Bearing Prosthesis during Dynamic Knee Extensor: A Case Trial
}

\author{
Takuya Osada1,2*, Masahiro Ishiyama1, Ryuichi Ueno ${ }^{1}$ \\ ${ }^{1}$ Rehabilitation Center, Tokyo Medical University Hospital, Tokyo, Japan \\ ${ }^{2}$ Cardiac Rehabilitation Center, Tokyo Medical University Hospital, Tokyo, Japan \\ Email: ^dentacmac@aol.com
}

How to cite this paper: Osada, T., Ishiyama, M. and Ueno, R. (2019) Voluntary Thigh Muscle Strength with Resection Stump-Dependent Blood Flow and Vasodilation in an Amputated Lower Leg with Total Surface Bearing Prosthesis during Dynamic Knee Extensor: A Case Trial. Open Journal of Therapy and Rehabilitation, 7, 151-169.

https://doi.org/10.4236/ojtr.2019.74011

Received: October 17, 2019

Accepted: November 12, 2019

Published: November 15, 2019

Copyright $\odot 2019$ by author(s) and Scientific Research Publishing Inc. This work is licensed under the Creative Commons Attribution International License (CC BY 4.0).

http://creativecommons.org/licenses/by/4.0/

\begin{abstract}
Background: The magnitude of the hyperemic response due to repeated thigh stump exercise on incremental contraction intensity might be useful information in localized exercise tolerance for devising cardiovascular physical therapy for amputees. The effect of exercise on amputated leg blood flow (LBF) may potentially be altered due to voluntary muscle contractions after loss of the lower leg compared with the healthy leg. Case Presentation: A 57-year-old male patient with Burger disease attempted 3 min unilateral repeat/dynamic knee extensor exercise at a target muscle contraction frequency (1 $\mathrm{s}$ thigh muscle contraction and $1 \mathrm{~s}$ relaxation, 90 repetitions) with each leg $<$ right transtibial amputated leg (AL) using a total surface-bearing prosthesis (TSB) and left non-AL $>$ at six different contraction intensities (rubber resistance belt). Simultaneous measurement of blood velocity/flow (Doppler ultrasound) in the femoral artery, blood pressure, leg vascular conductance (LVC), and peak muscle strength (PMS) were performed during the $3 \mathrm{~min}$ exercise period. The maximum voluntary contraction by one-legged isometric knee muscle contraction was $14.7 \mathrm{~kg}$ in non-AL and $7.9 \mathrm{~kg}$ in the AL with prosthesis. The relative PMS was defined as "PMS/maximum voluntary contraction $\times 100$ (\%)". Pre-exercise LBF was lower in the AL $(200 \pm 25 \mathrm{ml} / \mathrm{min})$ than the non-AL $(275 \pm 74 \mathrm{ml} / \mathrm{min})$. Both the non-AL and AL showed good positive linear relationships between absolute-/relative-PMS and LBF or LVC during $30 \mathrm{~s}$ at steady-state before the end of the exercise period. Furthermore, there was also similarity seen in the increase rate in LBF and/or LVC for the incremental relative PMS compared with the absolute PMS. Conclusion: In
\end{abstract}


this case, the muscle strength depended on blood flow increase/vasodilation was seen in this "AL" using a TSB prosthesis for repeated dynamic knee extensor exercise. The present amputee's limb muscle strengthening with the resection stump closely related to the degree of hyperemia in the amputated limb.

\section{Keywords}

Exercising Leg Blood Flow, Vasodilation, Transtibial Amputation, Total Surface Bearing Prosthesis, Doppler Ultrasound

\section{Introduction}

For leg amputees, physical and exercise therapy can have important roles in promoting activities of daily living for the prevention of joint contracture and walking disability [1] [2] [3] [4]. Therefore, it may be acceptable that motor function such as the joint range of motion in the knee/hip and improving muscle strength to benefit the metabolic cost of walking are high priorities when using a prosthesis [5]-[10].

Recently, the cause of leg amputation is most often due to the ischemia following peripheral vascular disease and/or microcirculatory disorder based on arteriosclerosis with diabetes mellitus, peripheral vascular disease, or Burger's disease, which may potentially limit the blood flow/oxygen supply feeding the vascular bed for the needs of the hyperemic state in exercise [11]. Consequently, dysfunction of the hyperemic state in vascular disease may be seen in limb skeletal muscle [12].

There is still a lack of understanding of thigh-stump exercise blood flow and/or vasodilation in relation to amputated limb muscle activity, nevertheless slight leg passive motion and/or active voluntary muscle contraction could initiate an increase in muscle metabolism with vasodilation relating to "leg oxygen uptake".

Therefore, blood flow may be a more important concern when prescribing exercise for amputees with potentially reduced exercise tolerance [13].

As part of cardiovascular rehabilitation, muscle blood flow stimuli due to physical activity/aerobic exercise may have a major role in oxygen transport for muscle metabolism in the limb, which is closely related to "exercise tolerance corresponding to systemic maximum oxygen uptake" [14] and/or "muscle strength power" [15]. During dynamic knee extensor exercise, increased leg oxygen uptake will be directly proportional to the work performed in the muscle [16] [17].

Oxygen uptake by the leg is theoretically calculated as the product of "arterial blood flow in the working leg" and the arteriovenous oxygen difference in the exercising leg. Thus, an evaluation of leg blood flow (LBF) dynamics feeding the contracting major thigh muscles to the incremental workload can contribute to 
understanding the muscle blood flow supply and vasodilation due to exercise and circulatory factors limiting work capacity in the thigh stump of the amputated leg (AL).

Ultrasound Doppler devices can provide high temporal resolution of blood velocity. Pulsatile blood velocity profile in the conduit artery at systole and diastole may be detected at rest, synchronized with the cardiac beat and blood pressure [18] [19] [20]. Based on this technique, rapid changes in blood velocity can be measured with muscle contraction and relaxation and/or cardiac beat-to-beat in different states of exercise, muscle contraction time/frequency and workload, and in relation to vasodilatation/vasoconstriction [21] [22]. In previous reports from healthy legs on exercise LBF measured by Doppler ultrasound, there were positive linear correlations between unilateral LBF and workload during steadystate rhythmic unilateral leg exercise [17] [23] [24] [25] [26].

Using the above-mentioned Doppler technique, we have recently reported a clinical intervention for LBF magnitude in an amputated lower leg with a patella tendon bearing (PTB) prosthesis during unilateral dynamic knee extensor exercise at incremental exercise intensity [27]. This initial brief report involved an unexpected result with no significant thigh LBF increases in the AL using a PTB prosthesis during incremental absolute/relative peak muscle contraction (workload), although workload-dependent LBF increases were seen in the non-AL, which was in agreement with previous findings.

It is still necessary, however, to investigate the time course of the magnitude of thigh stump LBF in amputees and/or using another prosthesis such as a widely known total surface-bearing (TSB) prosthesis during exercise.

Thus, a present case was preliminary clinical intervention trial with a TSB prosthesis in an attempt to measure exercising limb circulatory response in patients with an $\mathrm{AL}$ or non-AL in order to understand whether there is a close relationship between LBF/vasodilation and muscle contraction strength in the AL.

\section{Case Presentation}

\subsection{Participant}

A male $(57 \mathrm{yr} 8 \mathrm{mo}, 168.2 \mathrm{~cm}, 57.4 \mathrm{~kg}$ ) with trans-tibial amputation of the right lower leg due to Burger's disease and diabetes (at age $53 \mathrm{yr} 6 \mathrm{mo}$ ) participated in the study. The left ankle-brachial index was 1.13 . The length of the resected stump was $18.0 \mathrm{~cm}$ from the knee joint space to the stump-end, which supported walking using a TSB prosthesis. The weight of the TSB was $1.4 \mathrm{~kg}$. The range of knee angle motion in the AL was maintained for activities of daily living using the TSB. The circumference of the thigh was $45.5 \mathrm{~cm}$ at maximum, $39.8 \mathrm{~cm}$ at 10 $\mathrm{cm}$ above the patella, and $36.2 \mathrm{~cm}$ at $5 \mathrm{~cm}$ above the patella in the non-AL, and $44.5 \mathrm{~cm}$ at maximum, $34.6 \mathrm{~cm}$ at $10 \mathrm{~cm}$ above the patella, and $36.5 \mathrm{~cm}$ at $5 \mathrm{~cm}$ above the patella in the AL. The length between the greater trochanter and the knee joint space was $39 \mathrm{~cm}$ in both legs. The lower leg length was $38 \mathrm{~cm}$ in the non-AL. His cardiovascular condition was well controlled. The study was con- 
ducted in accordance with the principles of the Declaration of Helsinki (1964) and with approval of the Institutional Ethics Committee of the authors' institution (approval No. 2016-080). The participant gave written consent and was informed for the nature and purpose of the study and for further publication, as well as potential risks and discomfort. The participant was informed that withdrawal from the study was possible at any time without consequences.

\subsection{Exercise Model}

The rhythmic knee extensor exercise model used in the present case allowed for stable and validated measurements of blood velocity in the conduit femoral artery above the bifurcation using Doppler ultrasound [17] [23] [24] [28] (Figure 1). The knee extensor quadriceps muscle group was used to represent the activation of the large thigh muscle group, and previous research on LBF in relation to dynamic or static knee extensor exercise during incremental contraction power output has contributed to the evaluation of the magnitude of thigh muscle contraction-induced blood flow [18] [19] [23] [24] [28] [29]. The LBF value using an invasive thermodilution method obtained in similar experimental conditions by Andersen et al. [14] was similar to those obtained by non-invasive Doppler ultrasound [17].

\subsection{Study Protocol}

The participant's thigh was positioned horizontally, with the knee joint bent (90 degree flexion) in a sitting position (Figure 1). The maximum voluntary contraction (MVC) in the isometric knee extensor was measured using a strain gauge (see the section on MVC measurement) attached to each leg.

The participant was familiarized with kicking with his toe to reach a target point corresponding to 70 degree flexion from 90 degree flexion following the pace of a metronome as a target muscle contraction frequency before the test. The required range of motion in the knee angle was only 20 degrees, which was considered to be appropriate for stable repeated knee extensor exercise with a TSB for $3 \mathrm{~min}$.

Following $1 \mathrm{~min}$ of pre-exercise, the participant performed $3 \mathrm{~min}$ of unilateral repeat/dynamic knee extensor exercise at the target muscle contraction frequency of $<1 \mathrm{~s}$ muscle contraction (active knee extension) and $1 \mathrm{~s}$ relaxation (passive knee flexion) following an audible metronome, for a total of 90 repetitions with each leg (right AL using the TSB or left non-AL) at six different contraction strengths using rubber resistance bands while in the sitting position. Then, the participant had an at least $5-7$-min recovery phase after the end of each exercise session.

The absolute value for muscle contraction strength due to repeated knee extension was displayed in real time on a monitor connected to the strain gauge and amplifier.

The muscle contraction strength was adjusted using thin, medium, heavy, extra 


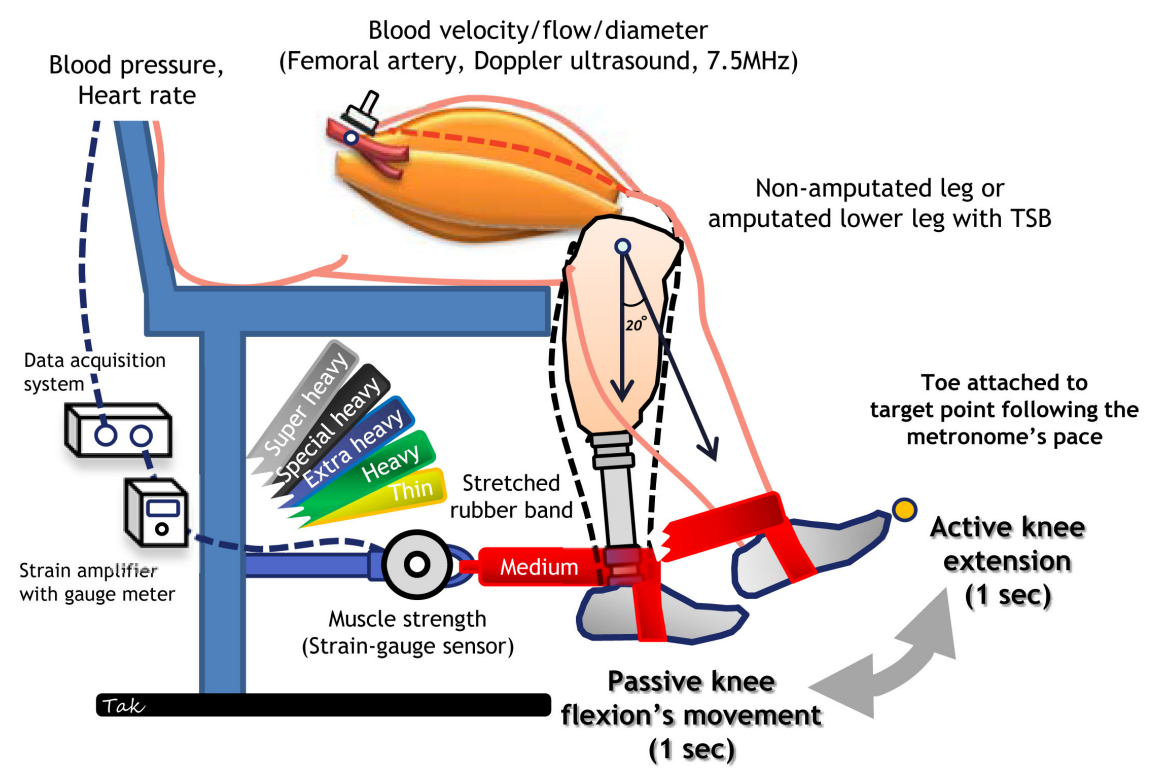

$<$ Experimental protocol>

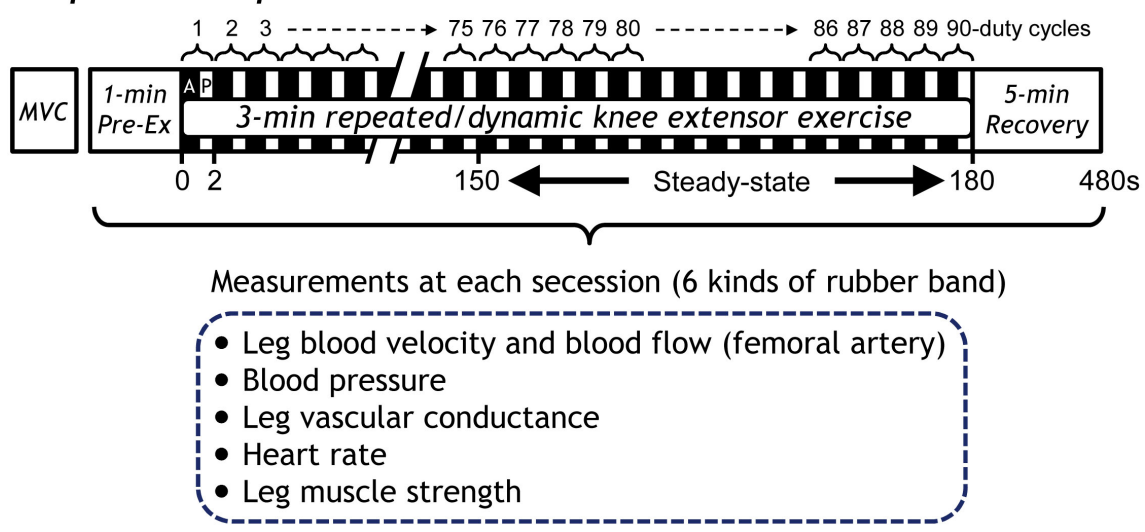

Figure 1. Scheme of one-legged dynamic knee extensor exercise and study protocol. Prior to the experiment, maximum voluntary contraction (MVC) was measured. A unilateral repeated/dynamic knee extensor exercise was performed at the target knee extensor frequency [1 s dynamic-active thigh muscle contraction and $1 \mathrm{~s}$ passive muscle relaxation (passive flexion movement): 90-duty cycles] for $3 \mathrm{~min}$ using six different rubber resistance bands on each leg following 1 min pre-exercise (Pre-Ex). The right amputated lower leg performed the exercise using a total surface bearing (TSB) prosthesis. Kicking with the toe was directed at a target point corresponding to 70-degree flexion of the knee joint angle from 90-degree flexion (knee angle motion: 20 degrees) in time with the pace of an audible metronome at the target muscle contraction frequency. The recovery phase took $7 \mathrm{~min}$ after the end of each exercise session. Blood velocity in the femoral artery (Doppler ultrasound) was measured continuously at pre-exercise, during exercise, and in recovery. Blood flow was calculated as the product of cross-sectional area and blood velocity. Simultaneous recording of muscle strength (strain-gauge sensor), blood pressure, leg vascular conductance (blood flow/blood pressure), and heart rate was also performed via the data acquisition system. A: active knee extension, P: passive flexion movement.

heavy, special heavy, and super heavy rubber bands (see the section on rubber bands). The recovery time was sufficient for the hemodynamic parameters to return to the resting control levels between exercise sessions. The parameters 
(blood velocity, blood pressure, heart rate, and muscle contraction strength) were recorded simultaneously at pre-exercise, during exercise, and in recovery. Steady-state during exercise was defined from $150 \mathrm{~s}$ to $180 \mathrm{~s}$ (76 - 90th duty kicking cycles) for the evaluation of peak muscle strength-dependent increases in $\mathrm{LBF}$ and vasodilation.

\subsection{Maximum Voluntary Contraction (MVC)}

Before starting the experiment, the MVC was measured as the maximum muscle contraction strength throughout a unilateral knee extensor isometric muscle contraction of each leg with the subject's thigh positioned horizontally and the knee joint bent (90 degree flexion) in the sitting position, in accordance with the previously validated procedure [27] [29].

MVC (in kg) was determined from the average of three repeated measurements using a strain gauge connected to a strain amplifier and gauge meter (Meiko Co. Ltd, Tokyo, Japan) and was recorded continuously on a computer using a PowerLab data acquisition system (Chart v.4.2.3 software; ADInstruments, Sydney, Australia) (Figure 1). The MVC was defined as the peak value of the muscle contraction strength curve profile. The peak muscle strength during exercise was also evaluated using the relative (percentage of) MVC (peak muscle strength/MVC $\times 100, \%)$.

\subsection{Voluntary Muscle Contraction Strength Using Rubber Bands}

Six different TheraBands ${ }^{\circledR}$ were used to test muscle contraction strength. The stiffness of each band was previously validated by the manufacturer [30] [31] [32]: thin (yellow, $1.3 \mathrm{~kg}$ ), medium (red, $1.7 \mathrm{~kg}$ ), heavy (green, $2.1 \mathrm{~kg}$ ), extra heavy (blue, $2.6 \mathrm{~kg}$ ), special heavy (black $3.3 \mathrm{~kg}$ ), and super heavy (silver, $4.6 \mathrm{~kg}$ ), the values of which formally represent the degree of strength required to stretch the rubber band $30 \mathrm{~cm}$ to $60 \mathrm{~cm}$ in the TheraBand ${ }^{\circledR}$ product information [33].

The peak muscle contraction strength (in $\mathrm{kg}$ ) of every knee extensor kick was evaluated using a strain gauge and amplifier [27]. The rubber band was tied in a loop, enclosing the ankle, with a sensor fixed in the chair strut connected to the strain gauge with amplifier (Meiko Co. Ltd, Tokyo, Japan), and values were recorded continuously on a computer using a PowerLab data acquisition system (Figure 1). The peak muscle contraction strength was defined as the peak value at the maximum amplitude of the muscle contraction strength curve (muscle contraction-relaxation cycle) during active knee extensor kicking via the acquisition system.

\subsection{Blood Velocity and Diameter in the Femoral Artery}

The high temporal resolution of Doppler ultrasound enables the continuous measurement of blood velocity (a time- and space-averaged and amplitudeweighted "mean blood velocity") in the conduit femoral artery during knee extensor exercise [17] [18] [19] [20] [23] [24] [28] [29] [34] [35]. 
The beat-to-beat blood velocity profile in the femoral artery was measured continuously using a 7.5 MHz pulsed Doppler ultrasound (GE Logiq 3, Tokyo, Japan) with a videotape recorder (AG-7350-P, Panasonic, Tokyo, Japan). The coefficient of variation $(<5 \%)$ for the repeated blood velocity measurements represented the criteria for quality control of the operator's technique (first author) at pre-exercise as well as during exercise [18] [19] [23] [24] [28] [29] [35]-[44].

The mean femoral arterial vessel diameter (distance between the proximal and distal intima in the artery) at the pulsatile diastolic phase for each beat was calculated over approximately five beats.

The value of the pre-exercise vessel diameter was used to calculate the femoral arterial LBF at pre-exercise, during one-legged repeated dynamic knee extensions, and in recovery, because the diameter does not significantly vary between pre-exercise and knee extensor exercise [23] [36] [45] [46].

LBF was calculated as the product of blood velocity and the cross-sectional area, which has been validated previously and shown to produce accurate absolute values both at rest and during rhythmical/dynamic thigh muscle contractions [17] [18] [19] [20] [23] [24] [28] [29].

\subsection{Blood Pressure and Heart Rate}

Blood pressure and heart rate were measured simultaneously using an auricular plethysmography device with oscillometric calibration, through a cuff tourniquet placed on the upper right arm (RadiaPress RBP-100, KANDS, Aichi, Japan). These values and the muscle contraction strength curve (muscle contractionrelaxation phase) from the stretched rubber band with strain-gauge connection, strain amplifier, and gauge meter (Meiko Co. Ltd., Tokyo, Japan) were recorded continuously on a computer using a PowerLab data acquisition system (Chart v.4.2.3 software; ADInstruments, Sydney, Australia) with 1 min pre-exercise, during a $3 \mathrm{~min}$ exercise period, and in $5 \mathrm{~min}$ recovery (Figure 1). The mean values of blood pressure and heart rate (defined as R-R interval of blood pressure curve) were extracted at the same time as the determinants of beat-by-beat blood velocity value.

\subsection{LBF and Leg Vascular Conductance (LVC)}

The time- and space-averaged and amplitude-weighted "mean blood velocity" in the femoral artery was measured by automatically by averaging the separate variables in each cardiac cycle. LBF in the femoral artery was calculated by multiplying the cross-sectional area [area $=\pi \times(\text { pre-exercise vessel diameter } / 2)^{2}$ ] by the mean the blood velocity at pre-exercise, during exercise, and in recovery. The LVC was calculated as LBF divided by blood pressure (LBF/blood pressure) using the unit $\mathrm{ml} / \mathrm{min} / \mathrm{mmHg}$.

\subsection{Evaluations and Statistics}

Mean LBF, blood velocity, blood pressure, LVC, and heart rate were measured as 
the average of every $30 \mathrm{~s}$ from the start of exercise ( $\mathrm{t}=0$ in figures), pre-exercise, during $3 \mathrm{~min}$ of exercise, and in the 7-min recovery period.

Statistical comparisons with a linear fitting regression correlation coefficient (r), and p-value were conducted between mean LBF and mean LVC, and the mean peak muscle contraction strength (relative value as \%MVC) at steady-state for the $30 \mathrm{~s}$ period before the end of exercise was examined. Furthermore, the slope (corresponding to the increase ratio of LBF to absolute- or relative-muscle contraction strength) in the regression line was also determined (Microsoft Excel 2010). A p-value $<0.05$ was considered significant. All values are mean \pm standard deviation $(\mathrm{SD})$.

\section{Results}

The MVC was $7.9 \mathrm{~kg}$ in the AL and $14.7 \mathrm{~kg}$ in the non-AL. The stability of muscle contraction cycle, peak muscle strength, and hemodynamic variables during each knee extension during $30 \mathrm{~s}$ steady-state exercise are shown in Table 1. During exercise, the peak muscle strength for each kick was stable throughout the 3-min exercise period, and steady-state with the coefficients of variations below $5 \%$ in Figure 2. The heart rate and mean blood pressure during steady-state

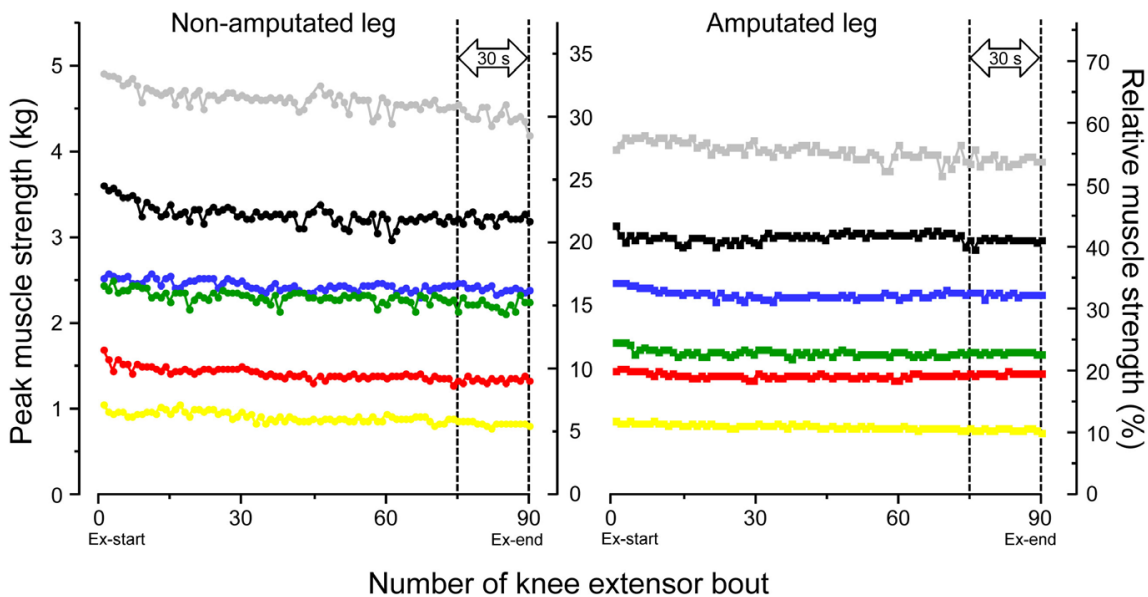

Figure 2. The fluctuation for peak muscle contraction in each knee extension during 3 min exercise. The peak muscle strength due to each knee extensor muscle contraction presented from the onset (Ex-start) to the end of exercise (Ex-end). The magnitude of peak muscle strength may indicate stable and repeated voluntary kicking for $3 \mathrm{~min}$ at the target muscle strength (see Table 1). In the non-amputated leg, the peak muscle strength had relatively large variability (describes as the exponential decay) during $30 \mathrm{sec}$ from the onset of exercise with the black (special heavy) and silver (super heavy) resistance band. The less fluctuation in peak muscle strength during $30 \mathrm{~s}$ steady-state $(\Leftrightarrow)$ before the end of exercise was in an acceptable range for the determination of (peak) muscle contraction strength-dependent blood flow increase and vasodilation. The maximum voluntary contraction (MVC) was different between the non-amputated $(14.7 \mathrm{~kg})$ and amputated lower legs $(7.9 \mathrm{~kg})$; therefore, the scale for the relative muscle strength (\%MVC, defined as peak muscle strength/MVC $\times 100, \%)$ is also shown as a vertical axis on the right side. Circles: non-amputated leg (non-AL). Squares: amputated leg (AL). The plotted data in various colors correspond to the resistance band colors-yellow (thin), red (medium), green (heavy), blue (extra heavy), black (special heavy) and silver (super heavy). 


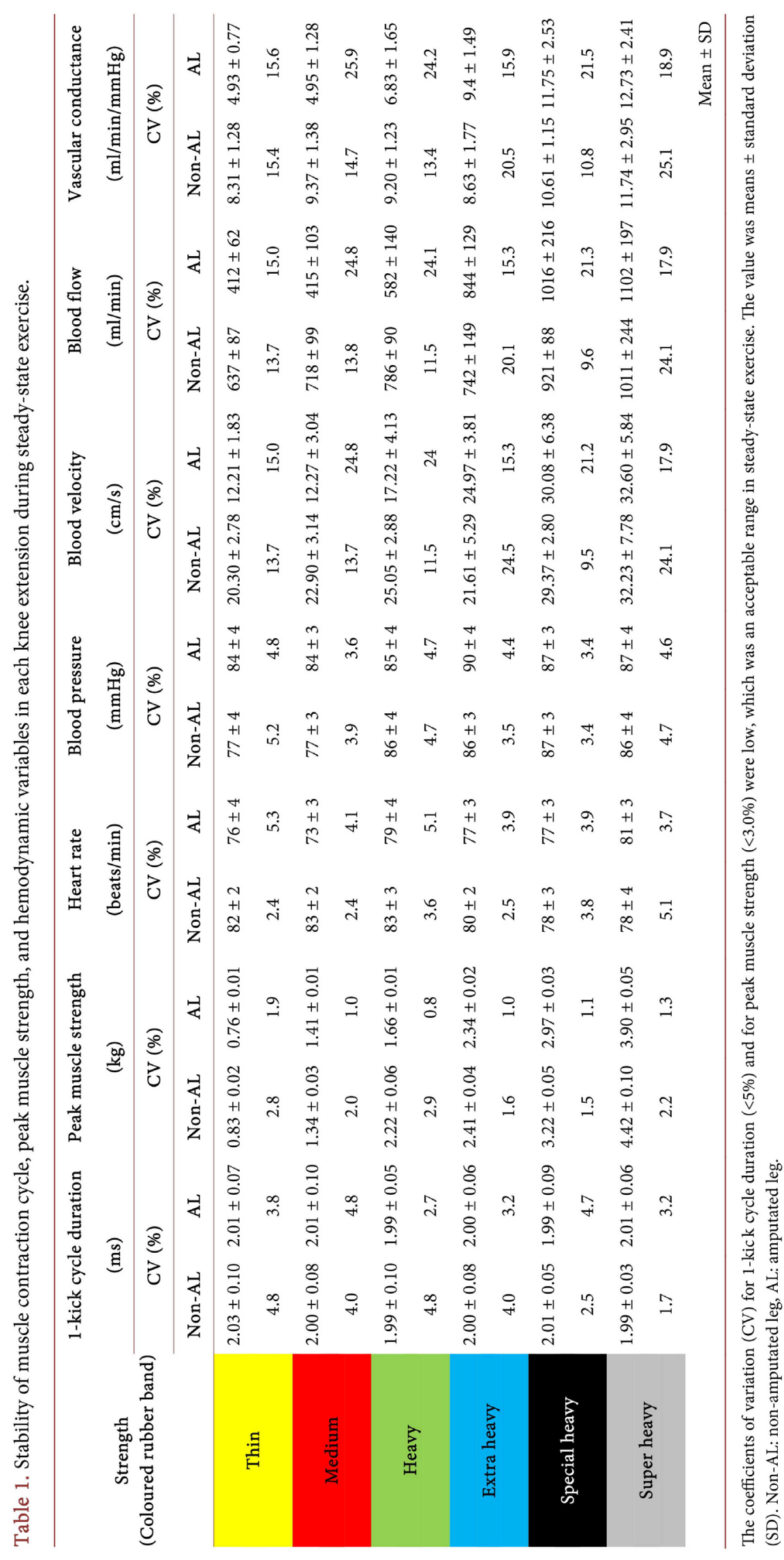


are shown in Figure 3. There was a significant positive linear relationship between "peak muscle contraction strength" and "LBF" at steady-state in the non-AL $(r=0.973, p<0.01$, regression line slope $=104.5)$ as well as the AL $(r=$ $0.963, p<0.01$, regression line slope $=255.9)$. Furthermore, the slope in the regression line for the relationship between "relative muscle contraction strength (\%MVC)" and "LBF" may be close between the AL (20.2) and non-AL (15.4) in Figure 4.

Regarding LVC, there was a significant positive linear relationship between "peak muscle contraction strength" and "LVC" at steady-state in the non-AL (r $=0.895, \mathrm{p}<0.05$, and regression line slope $=0.90)$ as well as $\mathrm{AL}(\mathrm{r}=0.967, \mathrm{p}<$ 0.01 , regression line slope $=2.88$ ). Furthermore, the slope in the regression line for the relationship between "relative muscle contraction strength" and "LVC" was close between the AL (0.23) and non-AL (0.13) in Figure 5.

\section{Discussion}

The present case may potentially indicate that muscle strength dependent-LBF and/or -vasodilation was also seen in a "below-knee amputation of the AL using TSB prosthesis" as well as in the healthy leg (non-AL) in repeated dynamic knee extensor exercise. This work is series in our previous case, which involved determination of the time course of magnitude in whole LBF in the exercising thigh stump using a PTB [27]. These findings are discussed in the following section.

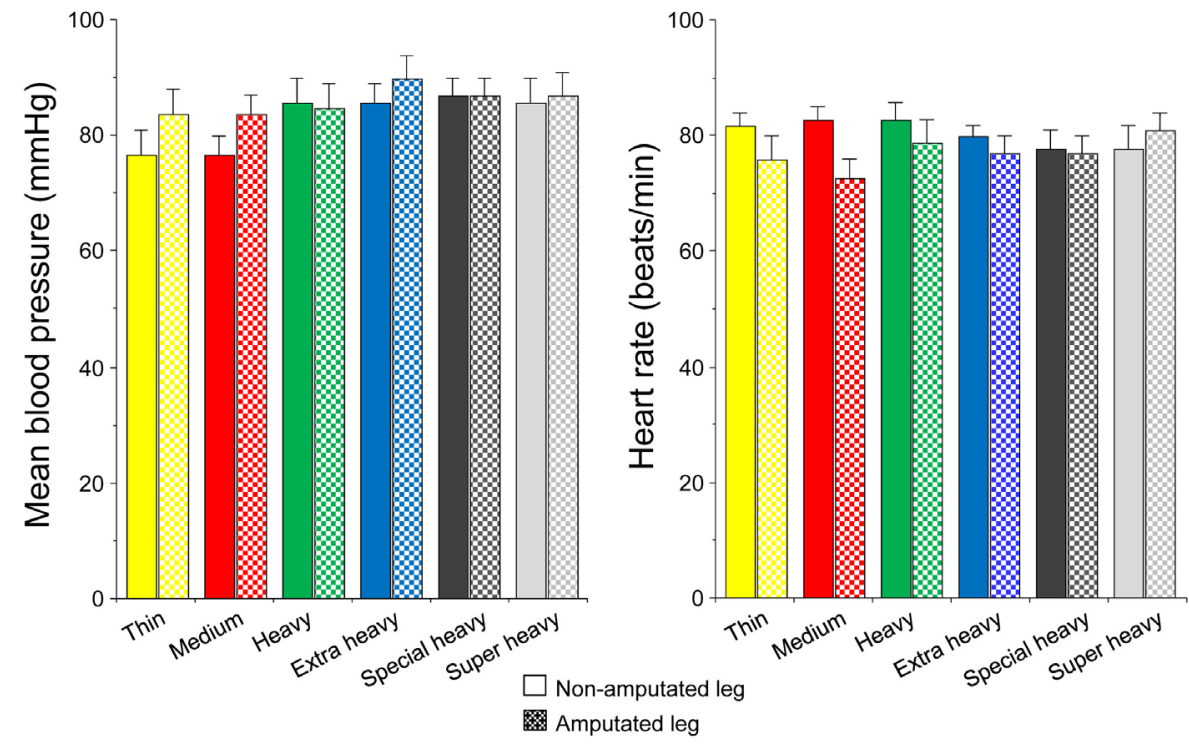

Figure 3. Mean blood pressure and heart rate in steady-state exercise. Mean blood pressure (MBP) tended to increase with an increase in muscle strength both legs except the silver band (super heavy). The MBP was similar between the green band (heavy) and blue band (extra heavy) in the non-amputated leg, and was also similar between the red band (medium) and green band (heavy) in the amputated leg. These similarities may be due to the smaller differences in peak muscle strength presented in Figure 2. The bars in various colors correspond to the resistance band colors. 

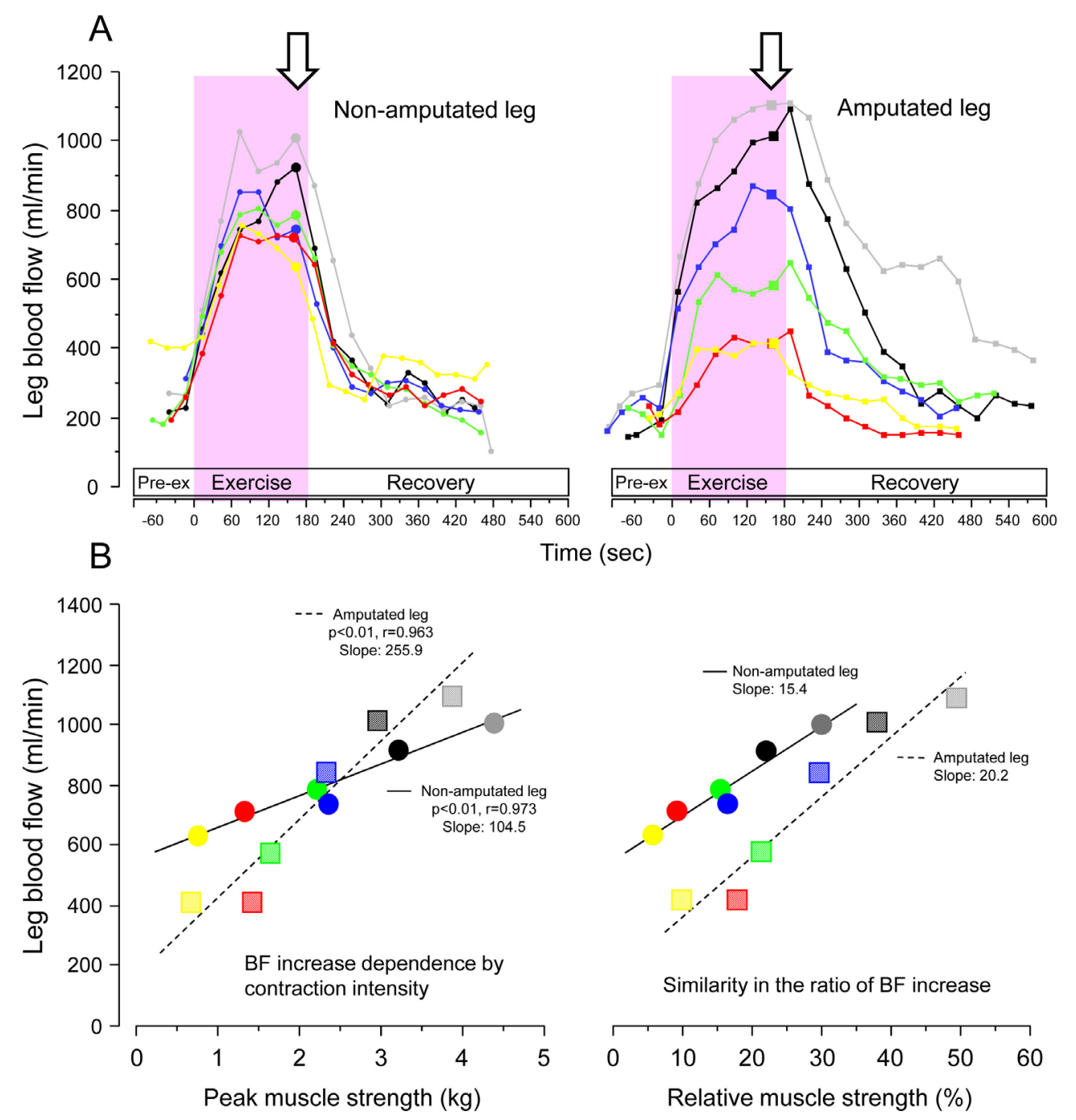

Figure 4. The time-course of LBF (A) and relation to absolute and relative muscle strength (B). The data were determined by averaging (net) every $30 \mathrm{~s}$. The net-leg blood flow (LBF) value for the last 30 $s$ of steady-state exercise ( $\downarrow$ in A) was used for the correlation between LBF and peak muscle strength/percentage of maximum voluntary contraction (relative muscle strength) as exercise intensity (B). Circles: non-amputated leg (non-AL). Squares: amputated leg (AL). The data plotted in various colors correspond to the resistance band colors-yellow (thin), red (medium), green (heavy), blue (extra heavy), black (special heavy) and silver (super heavy).

\subsection{LBF during Thigh Stump Exercise}

It is generally acknowledged that an increase in exercising LBF is directly proportional to the steady-state workload performed in relation to the interplay between cardiovascular regulation and muscle metabolism [17] [18] [23] [28]. The blood velocity and flow in the femoral artery increase linearly with incremental exercise intensities of work rate (for instance, peak muscle force) during steady-state rhythmic thigh muscle contractions (knee extensor exercise) [23] [24] [28]. This implies that enhanced vasodilatation is elicited, in relation to the increased average muscle force exerted at higher workloads, to meet the elevated metabolic activity. 

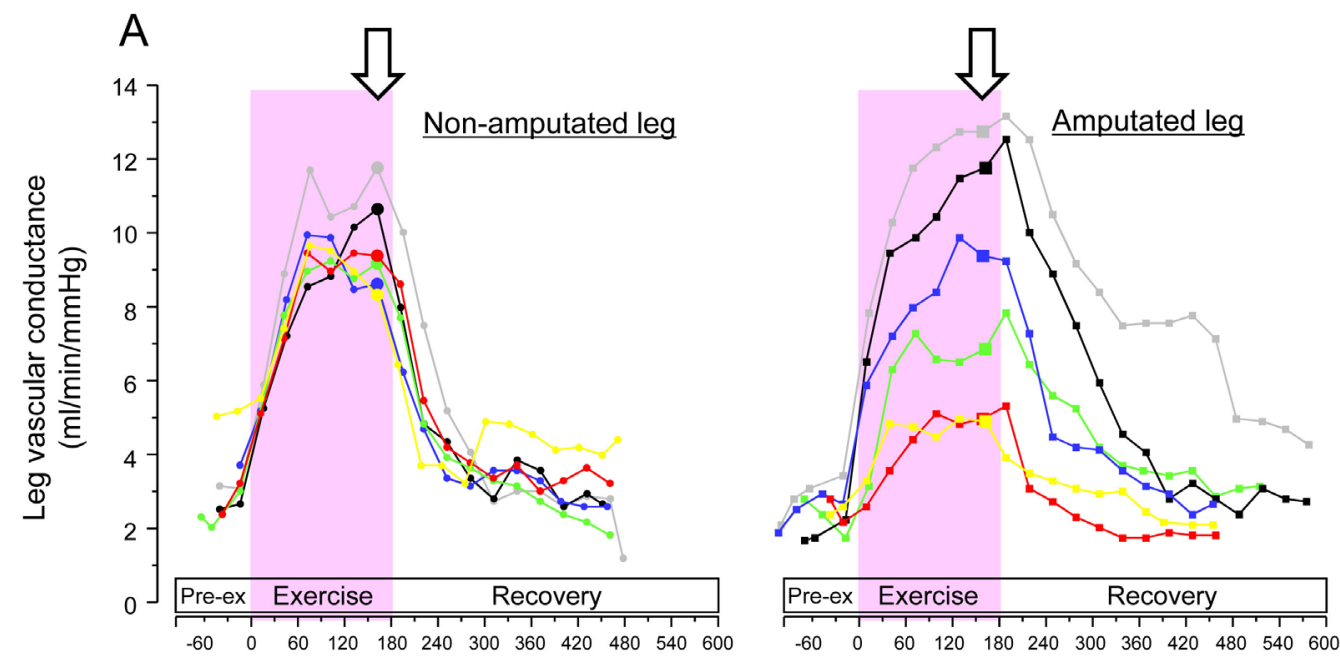

$\mathrm{B}$

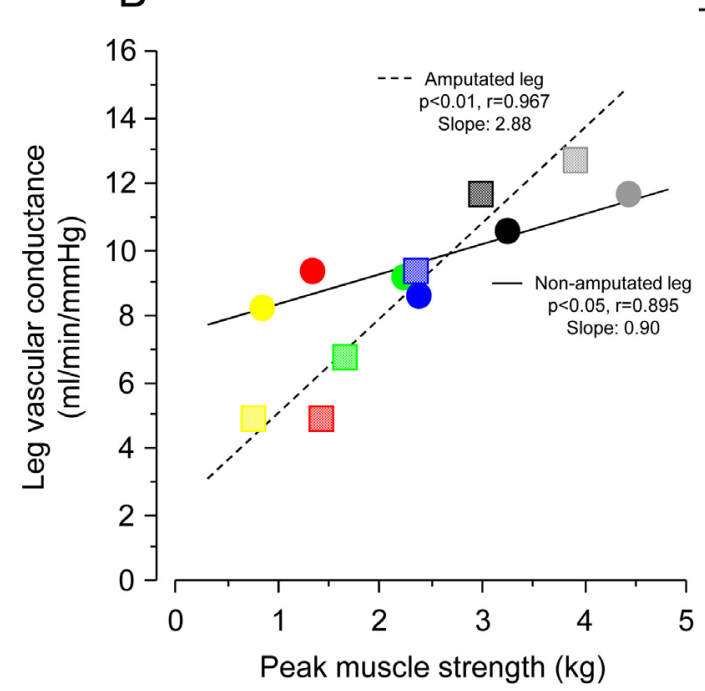

Time (sec)

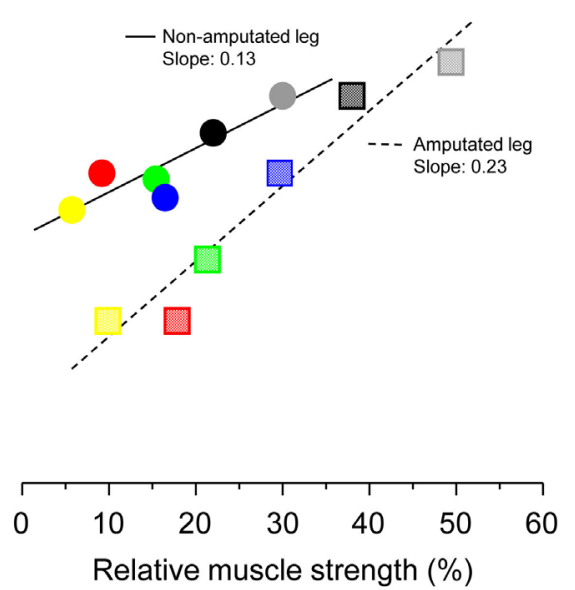

Figure 5. The time-course of LVC (A) and relation to absolute and relative muscle strength (B). The data were determined by averaging (net) every $30 \mathrm{~s}$. The net-leg vascular conductance (LVC) value for the last $30 \mathrm{~s}$ of steady-state exercise ( $\downarrow$ in A) was used for the correlation between leg vascular conductance and peak muscle strength/percentage of maximum voluntary contraction (relative muscle strength) as exercise intensity (B). Circles: non-amputated leg (non-AL). Squares: amputated leg (AL). The data plotted in various colors correspond to the resistance band colors-yellow (thin), red (medium), green (heavy), blue (extra heavy), black (special heavy) and silver (super heavy).

Thus, in the present case leg exercise induced LBF increase and/or leg vasodilation (corresponding to LVC) in left leg in the non-AL with the normal range of ankle-brachial index may be valid during steady-state exercise with stable hemodynamic parameters (Table 1). Furthermore, the thigh stump-LBF and -LVC in the $\mathrm{AL}$ also depending on the peak muscle contraction strength at the steady-state thigh exercise in Figure 4 and Figure 5, is in agreement with our previous findings.

The exercising LBF in healthy subjects has been investigated previously for limiting factors of exercise tolerance. However, there have been few studies targeting exercising LBF for amputees with potentially limited fitness and lower 
muscle strength, even if there is a need to evaluate residual function in the cardiovascular system. In addition, it is not easy to compare thigh-stump contraction induced LBF dynamics among amputees because of differences in the time periods after amputation, complications, medicines, or physical activity.

We reported recently on an interventional study with another amputee who performed knee extensor exercise using the same protocol as the present study [27]. The previous finding included determination of validated measurements of exercising thigh-stump LBF and its time-course in the amputated leg with PTB prosthesis using Doppler ultrasound. Furthermore, our clinical target was to examine whether the degree of muscle contraction intensity in the thigh of the $\mathrm{AL}$ is closely related to LBF and the hyperemic state.

The above-mentioned data in another amputee showed no significant increase in thigh LBF in the AL with incremental workload (peak muscle strength) but a close correlation between them in the non-AL.

We speculated that this discrepancy between the AL and non-AL may be due to a mismatch of the workload-dependent LBF increase in the disused and/or atrophic thigh muscle-resection stump of the AL compared with the non-AL, which in the AL may be due to the remaining muscle contractile effort with a reduced arterial inflow and/or lack of venous return in relation to the arteriovenous pressure gradient and/or hydrostatic pressure through the lower leg [27].

Prior to this study, we hypothesized the difference in the resection stump muscle contraction-induced alterations/magnitude in LBF and/or vasodilation between the $\mathrm{AL}$ and non-AL during incremental muscle contraction intensity, because there is no doubting the lack of venous return from lower leg owing to its amputation.

In the present case, however, the exercising LBF and/or LVC in the working thigh muscle using an TSB prosthesis was closely $(\mathrm{p}<0.01)$ related to the peak muscle contraction strength in AL in Figure 4 and Figure 5. This may indicate that thigh-stump exercise, even though the lower leg does not exist, may have a function in increasing blood flow and muscle vasodilation due to the thigh muscle contraction intensity.

Basically, the knee extension uses only the knee extensors of the quadriceps muscle group, and it therefore may require less activity of the lower leg and consequently not increase feeding inflow into the lower leg in non-AL. Therefore, it is expected that the changes in LBF (the rate of increasing LBF) may be related to muscle contraction strength between $\mathrm{AL}$ and non-AL if only the thigh parts are active.

Of note, the rate of increasing both LBF and LVC, which correspond to the slope of the linear regression, may be in a similar range between the non-AL and $\mathrm{AL}$ in relative muscle contraction intensity (\%MVC) rather than absolute muscle contraction intensity (slope in LBF; non-AL vs. AL: 105 vs. 256 at absolute intensity $\rightarrow 15$ vs. 20 at relative intensity in Figure 4, slope in LVC; non-AL vs. AL: 0.9 vs. 2.9 at absolute intensity $\rightarrow 0.13$ vs. 0.23 at relative intensity in Figure 
5).

This data may potentially suggest that the LBF increase and/or vasodilation are precisely dependent on the relative intensity rather than absolute muscle strength. In the present case, the MVC in the AL $(8 \mathrm{~kg})$ was half of that in the non-AL $(15 \mathrm{~kg})$, consequently the muscle volume was smaller in the AL than the non-AL because the circumference was shorter in the AL than the non-AL at 10 $\mathrm{cm}$ above the patella, which may potentially indicate the presence of muscle atrophy in the AL. It can be considered that muscle strength training in the thigh stump has a significant role in muscle contraction-induced blood flow increases and/or vasodilation hyperemic state for amputees.

\subsection{Possible Explanation for the Difference in Exercising LBF by TSB and PTB Prosthesis}

There have been few investigations about the relationship of "resection stump muscle contraction exercise" between "LBF and/or vasodilation" in amputated lower legs. This might be the reason why there is difficulty in obtaining stable LBF measurement and evaluation during constant voluntary and rhythmic muscle contractions of the thigh-stump at a target workload. In addition, it may not be easy to compare the magnitude of $\mathrm{LBF}$ between the AL and non-AL during unilateral leg exercise because of dissimilarity in the exercise model without prosthesis due to loss of physiological/biomechanical function of the lower leg muscle mass, limitation in the range of motion of the knee joint, and/or stable load-setting for the thigh muscle strength.

In our previous report using PTB prosthesis, a below-knee amputee was able to perform repeated knee extensor movements with a possible comparison of LBF magnitude between the AL and non-AL [27].

It is, however, still unknown the how the prosthesis impacts on voluntary muscle contraction strength. The weakness of thigh stump muscle contraction-induced LBF increases present in the previous case [27] may be due to the lack of sufficient voluntary muscle force by the PTB prosthesis with characteristic lateral fluctuation of the knee joint [47]. Therefore, we speculated that there is less uniformity in muscle contraction (larger fluctuation of muscle tension during repeated kicking contraction) via the socket suspended by the cuff suspension strap on the AL.

To overcome the disadvantages of a PTB prosthesis, a TSB prosthesis has been widely used for the stability of suspension via a silicon liner with a pin-attachment locked-adapter, which can coordinate the precise muscle force-generated as an increase in muscle strength during voluntary thigh muscle contractions. A TSB prosthesis with vacuum-assisted suction sockets may improve gait symmetry, residual limb activity [48]; therefore, we assumed that a TSB prosthesis with good fixation by a socket-silicon linear connecting thigh-stump may improve functional voluntary thigh muscle force/power during knee extension compared with a PTB prosthesis. Consequently, it was seen that proper thigh-stump muscle contraction induced LBF increases through stability with thigh stump exercise. 


\section{Conclusion}

In the present interventional case as preliminary trial, we examined whether an amputee showed muscle strength dependent on increases in LBF/vasodilation in an "AL" using a TSB prosthesis during rhythmic muscle exercise. In the present amputee, limb muscle strengthening in the resected stump closely related with the degree of hyperemia in the amputated limb.

Only one case is insufficient for the conclusive evidence regarding the relationship between leg blood flow (LBF)/vasodilation and muscle contraction strength in the AL, thus further research would need a retrospective study and enroll more patients. However, cardiovascular rehabilitation may potentially include new insights into the importance of interactions between muscle strength and peripheral circulatory adjustment for patients with below-knee amputation as well as for chronic critical limb ischemia.

\section{Acknowledgements}

The first author acknowledges the long-term support of the late professor emeritus Bengt Saltin of The Denmark Copenhagen Muscle Research Centre as well as the late professor emeritus Hisao Iwane of formerly The Tokyo Medical College for contributions leading to the present state of clinical research in environmental exercise and applied physiology for rehabilitation. The data in this article were partially presented at the 56th Annual Meeting of the Japanese Association of Rehabilitation Medicine in 2019. The study was supported by a Scientific Research (C) general grant (No. 15K01730) from MEXT and JSPS (to T. Osada).

\section{Conflicts of Interest}

The authors declare that there is no conflict of interest associated with this work.

\section{References}

[1] Ülger, Ö., Şahan, T.Y. and Çelik, S.E. (2018) A Systematic Literature Review of Physiotherapy and Rehabilitation Approaches to Lower-Limb Amputation. Physiotherapy Theory and Practice, 34, 821-834. https://doi.org/10.1080/09593985.2018.1425938

[2] Lin, S.J., Winston, K.D., Mitchell, J., Girlinghouse, J. and Crochet, K. (2014) Physical Activity, Functional Capacity, and Step Variability during Walking in People with Lower-Limb Amputation. Gait \& Posture, 40, 140-144. https://doi.org/10.1016/j.gaitpost.2014.03.012

[3] Schafer, Z.A., Perry, J.L. and Vanicek, N. (2018) A Personalised Exercise Programme for Individuals with Lower Limb Amputation Reduces Falls and Improves Gait Biomechanics: A Block Randomised Controlled Trial. Gait \& Posture, 63, 282-289. https://doi.org/10.1016/j.gaitpost.2018.04.030

[4] Hordacre, B., Birks, V., Quinn, S., Barr, C., Patritti, B.L. and Crotty, M. (2013) Physiotherapy Rehabilitation for Individuals with Lower Limb Amputation: A 15-Year Clinical Series. Physiotherapy Research International, 18, 70-80.

https://doi.org/10.1002/pri.1529 
[5] Vanross, E.R., Johnson, S. and Abbott, C.A. (2009) Effects of Early Mobilization on Unhealed Dysvascular Transtibial Amputation Stumps: A Clinical Trial. Archives of Physical Medicine and Rehabilitation, 90, 610-617. https://doi.org/10.1016/j.apmr.2008.10.026

[6] Russell Esposito, E. and Miller, R.H. (2018) Maintenance of Muscle Strength Retains a Normal Metabolic Cost in Simulated Walking after Transtibial Limb Loss. PLoS ONE, 12, e0191310. https://doi.org/10.1371/journal.pone.0191310

[7] Nadollek, H., Brauer, S. and Isles, R. (2002) Outcomes after Trans-Tibial Amputation: The Relationship between Quiet Stance Ability, Strength of Hip Abductor Muscles and Gait. Physiotherapy Research International, 7, 203-214. https://doi.org/10.1002/pri.260

[8] Pedrinelli, A., Saito, M., Coelho, R.F., Fontes, R.B. and Guarniero, R. (2002) Comparative Study of the Strength of the Flexor and Extensor Muscles of the Knee through Isokinetic Evaluation in Normal Subjects and Patients Subjected to TransTibial Amputation. Prosthetics and Orthotics International, 26, 195-205. https://doi.org/10.1080/03093640208726648

[9] Renström, P., Grimby, G. and Larsson, E. (1983) Thigh Muscle Strength in Below-Knee Amputees. Scandinavian Journal of Rehabilitation Medicine, 9, 163-173.

[10] Nolan, L. (2012) A Training Programme to Improve Hip Strength in Persons with Lower Limb Amputation. Journal of Rehabilitation Medicine, 44, 241-248. https://doi.org/10.2340/16501977-0921

[11] Hussain, M.A., Al-Omran, M., Salata, K., Sivaswamy, A., Forbes, T.L., Sattar, N., Aljabri, B., Kayssi, A., Verma, S. and de Mestral, C. (2019) Population-Based Secular Trends in Lower-Extremity Amputation for Diabetes and Peripheral Artery Disease. Canadian Medical Association Journal, 191, E955-E961.

https://doi.org/10.1503/cmaj.190134

[12] Paula-Ribeiro, M., Garcia, M.M., Martinez, D.G., Lima, J.R. and Laterza, M.C. (2015) Increased Peripheral Vascular Resistance in Male Patients with Traumatic Lower Limb Amputation: One Piece of the Cardiovascular Risk Puzzle. Blood Pres sure Monitoring, 20, 341-345. https://doi.org/10.1097/MBP.0000000000000148

[13] Wezenberg, D., de Haan, A., Faber, W.X., Slootman, H.J., van der Woude, L.H. and Houdijk, H. (2012) Peak Oxygen Consumption in Older Adults with a Lower Limb Amputation. Archives of Physical Medicine and Rehabilitation, 93, 1924-1929. https://doi.org/10.1016/j.apmr.2012.05.020

[14] Andersen, P. and Saltin, B. (1985) Maximal Perfusion of Skeletal Muscle in Man. Journal of Physiology, 366, 233-249. https://doi.org/10.1113/jphysiol.1985.sp015794

[15] Bunout, D., Barrera, G., Hirsch, S., Jimenez, T. and de la Maza, M.P. (2018) Association between Activity Energy Expenditure and Peak Oxygen Consumption with Sarcopenia. BMC Geriatrics, 3, 298. https://doi.org/10.1186/s12877-018-0993-y

[16] Andersen, P., Adams, R.P., Sjøgaard, G., Thorboe, A. and Saltin, B. (1985) Dynamic Knee Extension as Model for Study of Isolated Exercising Muscle in Humans. Journal of Applied Physiology, 59, 1647-1653. https://doi.org/10.1152/jappl.1985.59.5.1647

[17] Rådegran, G. (1997) Ultrasound Doppler Estimates of Femoral Artery Blood Flow during Dynamic Knee Extensor Exercise in Humans. Journal of Applied Physiolo$g y$, 83, 1383-1388. https://doi.org/10.1152/jappl.1997.83.4.1383

[18] Osada, T. and Rådegran, G. (2006) Alterations in the Blood Velocity Profile Influence the Blood Flow Response during Muscle Contractions and Relaxations. Journal of Physiological Science, 56, 195-203. https://doi.org/10.2170/physiolsci.RP002905 
[19] Osada, T. and Rådegran, G. (2005) Alterations in the Rheological Flow Profile in Conduit Femoral Artery during Rhythmic Thigh Muscle Contractions in Humans. Japanese Journal of Physiology, 55, 19-28. https://doi.org/10.2170/jjphysiol.R2074

[20] Osada, T., Saltin, B., Mortensen, S.P. and Rådegran, G. (2012) Measurement of the Exercising Blood Flow during Rhythmical Muscle Contractions Assessed by Doppler Ultrasound: Methodological Considerations. Journal of Biomedical Science and Engineering, 5, 779-788. https://doi.org/10.4236/jbise.2012.512A098

[21] Osada, T. and Rådegran, G. (2016) Difference in Muscle Blood Flow Fluctuations between Dynamic and Static Thigh Muscle Contractions: How to Evaluate Exercise Blood Flow by Doppler Ultrasound. Physical Medicine and Rehabilitation Research, 1, 128. https://doi.org/10.15761/PMRR.1000128

[22] Osada, T., Saltin, B. and Rådegran, G. (2013) Assessment of Voluntary Rhythmic Muscle Contraction-Induced Exercising Blood Flow Variability Measured by Doppler Ultrasound. Open Journal of Molecular and Integrative Physiology, 3, 158-165. https://doi.org/10.4236/ojmip.2013.34021

[23] Osada, T. (2004) Muscle Contraction-Induced Limb Blood Flow Variability during Dynamic Knee Extensor. Medicine \& Science in Sports \& Exercise, 36, 1149-1158. https://doi.org/10.1249/01.MSS.0000132272.36832.6A

[24] Osada, T. and Rådegran, G. (2009) Femoral Artery Blood Flow and Its Relationship to Spontaneous Fluctuations in Rhythmic Thigh Muscle Workload. Clinical Physiology and Functional Imaging, 29, 277-292. https://doi.org/10.1111/j.1475-097X.2009.00868.x

[25] Shoemaker, J.K., Hodge, L. and Hughson, R.L. (1994) Cardiorespiratory Kinetics and Femoral Artery Blood Velocity during Dynamic Knee Extension Exercise. Journal of Applied Physiology, 77, 2625-2632. https://doi.org/10.1152/jappl.1994.77.6.2625

[26] Hughson, R.L., Shoemaker, J.K., Tschakovsky, M.E. and Kowalchuk, J.M. (1996) Dependence of Muscle VO2 on Blood Flow Dynamics at Onset of Forearm Exercise. Journal of Applied Physiology, 81, 1619-1626.

https://doi.org/10.1152/jappl.1996.81.4.1619

[27] Osada, T., Ishiyama, M. and Ueno, R. (2018) Time-Course of Thigh Muscle Contraction-Induced Blood Flow Magnitude in Amputated Lower Limb with Prosthesis during Dynamic Knee Extension: A Case Study. Physical Therapy and Rehabilitation, 5, 21. https://doi.org/10.7243/2055-2386-5-21

[28] Osada, T. and Rådegran, G. (2002) Femoral Artery Inflow in Relation to External and Total Work Rate at Different Knee Extensor Contraction Rates. Journal of Applied Physiology, 92, 1325-1330. https://doi.org/10.1152/japplphysiol.00848.2001

[29] Osada, T., Mortensen, S.P. and Rådegran, G. (2015) Mechanical Compression during Repeated Sustained Isometric Muscle Contractions and Hyperemic Recovery in Healthy Young Males. Journal of Physiological Anthropology, 34, 36.

https://doi.org/10.1186/s40101-015-0075-1

[30] Hughes, C.J., Hurd, K., Jones, A. and Sprigle, S. (1999) Resistance Properties of Thera-Band Tubing during Shoulder Abduction Exercise. Journal of Orthopaedic \& Sports Physical Therapy, 29, 413-420. https://doi.org/10.2519/jospt.1999.29.7.413

[31] Patterson, R.M., Stegink Jansen, C.W., Hogan, H.A. and Nassif, M.D. (2001) Material Properties of Thera-Band Tubing. Physical Therapy, 81, 1437-1445. https://doi.org/10.1093/ptj/81.8.1437

[32] Sakanoue, N. and Katayama, K. (2007) The Resistance Quantity in Knee Extension Movement of Exercise Bands (Thera-Band ${ }^{\circ}$ ). Journal of Physical Therapy Science, 19, 287-291. https://doi.org/10.1589/jpts.19.287 
[33] Thera-Band Tubing. Resistance Band \& Tubing, Instruction Manual. Resistance Performance Health Academy, Thera-Band Academy, Product Manuals, Directions for Handling, Connecting, and Securing TheraBand. http://www.thera-bandacademy.com/products/product-manuals.aspx

[34] Rådegran, G. and Saltin, B. (1998) Muscle Blood Flow at Onset of Dynamic Exercise in Humans. American Journal of Physiology, 274, H314-H322. https://doi.org/10.1152/ajpheart.1998.274.1.H314

[35] Osada, T. and Rådegran, G. (2006) Differences in Exercising Limb Blood Flow Variability between Cardiac and Muscle Contraction Cycle Related Analysis during Dynamic Knee Extensor. Journal of Sports Medicine and Physical Fitness, 46, 590-597.

[36] Osada, T., Katsumura, T., Hamaoka, T., Inoue, S., Esaki, K., Sakamoto, A., Murase, N., Kajiyama, J., Shimomitsu, T. and Iwane, H. (1999) Reduced Blood Flow in Abdominal Viscera Measured by Doppler Ultrasound during One-Legged Knee Extension. Journal of Applied Physiology, 86, 709-719.

https://doi.org/10.1152/jappl.1999.86.2.709

[37] Osada, T. (2012) Physiological Aspects of the Determination of Comprehensive Arterial Inflows in the Lower Abdomen Assessed by Doppler Ultrasound. Cardiovas cular Ultrasound, 10, 13. https://doi.org/10.1186/1476-7120-10-13

[38] Osada, T., Katsumura, T., Murase, N., Sako, T., Higuchi, H., Kime, R., Hamaoka, T. and Shimomitsu, T. (2003) Post-Exercise Hyperemia after Ischemic and NonIschemic Isometric Handgrip Exercise. Journal of Physiological Anthropology and Applied Human Science, 22, 299-309. https://doi.org/10.2114/jpa.22.299

[39] Osada, T., Murase, N., Kime, R., Shiroishi, K., Shimomura, K., Nagata, H. and Katsumura, T. (2007) Arterial Blood Flow of All Abdominal-Pelvic Organs Using Doppler Ultrasound: Range, Variability, and Physiological Impact. Physiological Measurement, 28, 1303-1316. https://doi.org/10.1088/0967-3334/28/10/014

[40] Osada, T., Nagata, H., Murase, N., Shimomura, K., Kime, R., Shiroishi, K., Nakagawa, N. and Katsumura, T. (2009) Hemodynamics Relationships among Upper Abdominal Aorta and Femoral Arteries: Basis for Measurement of Arterial Blood Flow to Abdominal-Pelvic Organs. Medical Science Monitor, 15, CR332-CR340.

[41] Osada, T., Nagata, H., Murase, N., Kime, R. and Katsumura, T. (2011) Determination of Comprehensive Arterial Blood Inflow in Abdominal-Pelvic Organs: Impact of Respiration and Posture on Organ Perfusion. Medical Science Monitor, 17, CR57-CR66. https://doi.org/10.12659/MSM.881388

[42] Osada, T., Katsumura, T., Hamaoka, T., Murase, N., Naka, M. and Shimomitsu, T. (2002) Quantitative Effects of Respiration on Venous Return during Single Knee Extension-Flexion. International Journal of Sports Medicine, 23, 183-190. https://doi.org/10.1055/s-2002-23177

[43] Osada, T. (2004) Exercise-Related Time Course of Pulsatility Index in Brachial Artery Following Forearm Exercise Assessed by Doppler Ultrasound. Tohoku Journal of Experimental Medicine, 203, 241-252. https://doi.org/10.1620/tjem.203.241

[44] Osada, T., Iwane, H., Katsumura, T., Murase, N., Higuchi, H., Sakamoto, A., Hamaoka, T. and Shimomitsu, T. (2012) Relationship between Reduced Lower Abdominal Blood Flows and Heart Rate in Recovery Following Cycling Exercise. Acta Physiologica, 204, 344-353. https://doi.org/10.1111/j.1748-1716.2011.02349.x

[45] Isnard, R., Lechat, P., Kalotka, H., Chikr, H., Fitoussi, S., Salloum, J., Golmard, J.-L., Thomas, D. and Komajda, M. (1996) Muscular Blood Flow Response to Submaximal Leg Exercise in Normal Subjects and in Patients with Heart Failure. Jour- 
nal of Applied Physiology, 81, 2571-2579.

https://doi.org/10.1152/jappl.1996.81.6.2571

[46] MacDonald, M.J., Shoemaker, J.K., Tschakovsky, M.E. and Hughson, R.L. (1998) Alveolar Oxygen Uptake and Femoral Artery Blood Flow Dynamics in Upright and Supine Leg Exercise in Humans. Journal of Applied Physiology, 85, 1622-1628. https://doi.org/10.1152/jappl.1998.85.5.1622

[47] Yiğiter, K., Sener, G. and Bayar, K. (2002) Comparison of the Effects of Patellar Tendon Bearing and Total Surface Bearing Sockets on Prosthetic Fitting and Rehabilitation. Prosthetics and Orthotics International, 26, 206-212. https://doi.org/10.1080/03093640208726649

[48] Safari, M.R. and Meier, M.R. (2015) Systematic Review of Effects of Current Transtibial Prosthetic Socket Designs Part 2: Quantitative Outcomes. Journal of Rehabilitation Research and Development, 52, 509-526.

https://doi.org/10.1682/JRRD.2014.08.0184 